\title{
Modelling and control of discrete event systems using switching max-plus-linear systems*
}

\author{
T.J.J. van den Boom and B. De Schutter
}

If you want to cite this report, please use the following reference instead:

T.J.J. van den Boom and B. De Schutter, "Modelling and control of discrete event systems using switching max-plus-linear systems," Proceedings of the 7th International Workshop on Discrete Event Systems (WODES'04), Reims, France, pp. 115-120, Sept. 2004.

Delft Center for Systems and Control

Delft University of Technology

Mekelweg 2, 2628 CD Delft

The Netherlands

phone: +31-15-278.24.73 (secretary)

URL: https: //www.dcsc.tudelft.nl

*This report can also be downloaded viahttps://pub.deschutter.info/abs/04_001.html 


\title{
MODELLING AND CONTROL OF DISCRETE EVENT SYSTEMS USING SWITCHING MAX-PLUS-LINEAR SYSTEMS
}

\author{
T.J.J. van den Boom ${ }^{1}$ and B. De Schutter ${ }^{1}$, \\ Delft Center for Systems and Control, Delft University of Technology, \\ Mekelweg 2, 2628 CD Delft, The Netherlands,
}

\begin{abstract}
In this paper we consider the modelling and control of discrete event systems using switching max-plus-linear systems. In switching max-plus-linear systems we can switch between different modes of operation. In each mode the discrete event system is described by a max-plus-linear state equation with different system matrices for each mode. The switching allows us to change the structure of the system, to break synchronization and to change the order of events. We will give some examples of this type of system. We define the model predictive control design problem for this type of discrete event system, and we show that solving this problem in general leads to a mixed integer optimization problem.
\end{abstract}

\section{INTRODUCTION}

The class of discrete event systems essentially consists of man-made systems that contain a finite number of resources (such as machines, communications channels, or processors) that are shared by several users (such as product types, information packets, or jobs) all of which contribute to the achievement of some common goal (the assembly of products, the end-to-end transmission of a set of information packets, or a parallel computation) [1]. In general, models that describe the behavior of a discrete event system are nonlinear in conventional algebra. However, there is a class of discrete event systems - the max-plus-linear discrete event systems - that can be described by a model that is "linear" in the max-plus algebra [1, 4], which has maximization and addition as its basic operations. The max-plus-linear discrete event systems can be characterized as the class of discrete event systems in which only synchronization and no concurrency or choice occurs.

In this paper we will consider discrete event systems that can switch between different modes of operation. In each mode the system is described by a max-plus-linear state equation with different system matrices for each mode. The switching changes the structure of the system, and so allows us to break synchronization and to change the order of events. We define a control design problem for such a system to optimize the system's behavior. In general this will lead to a mixed integer optimization problem.

\footnotetext{
${ }^{1}$ t.j.j.vandenboom, b. deschutter\}@dcsc.tudelft nl, http://www. dcsc.tudelft.nl
}

\section{MAX-PLUS ALGEBRA AND SWITCHING MAX-PLUS-LINEAR SYSTEMS}

\subsection{Max-plus algebra}

In this section we give the basic definition of the max-plus algebra and we present some results on a class of max-plus functions.

Define $\varepsilon=-\infty$ and $\mathbb{R}_{\varepsilon}=\mathbb{R} \cup\{\varepsilon\}$. The maxplus-algebraic addition $(\oplus)$ and multiplication $(\otimes)$ are defined as follows [1, 4]:

$$
x \oplus y=\max (x, y) \quad x \otimes y=x+y
$$

for numbers $x, y \in \mathbb{R}_{\varepsilon}$, and

$$
\begin{aligned}
& {[A \oplus B]_{i j}=a_{i j} \oplus b_{i j}=\max \left(a_{i j}, b_{i j}\right)} \\
& {[A \otimes C]_{i j}=\bigoplus_{k=1}^{n} a_{i k} \otimes c_{k j}=\max _{k=1, \ldots, n}\left(a_{i k}+c_{k j}\right)}
\end{aligned}
$$

for matrices $A, B \in \mathbb{R}_{\varepsilon}^{m \times n}$ and $C \in \mathbb{R}_{\varepsilon}^{n \times p}$.

\subsection{Max-plus-linear systems}

In $[1,4]$ it has been shown that discrete event systems in which there is synchronization but no concurrency can be described by a model of the form

$$
x(k)=A(k) \otimes x(k-1) \oplus B(k) \otimes u(k) .
$$

Systems that can be described by this model will be called max-plus-linear systems. The index $k$ is called the event counter. For discrete event systems the state $x(k)$ typically contains the time instants at which the internal events occur for the $k$ th time, the input $u(k)$ contains the time instants at which the input events occur for the $k$ th time, and the output $y(k)$ contains the time instants at which the output events occur for the $k$ th time. 


\subsection{Switching max-plus-linear systems}

In this paper we will consider discrete event systems that can switch between different modes of operation. In each different mode $\ell=1, \ldots, n_{\mathrm{m}}$, the system is described by a max-plus-linear state equation

$$
x(k)=A^{(\ell)}(k) \otimes x(k-1) \oplus B^{(\ell)}(k) \otimes u(k)
$$

in which the matrices $A^{(\ell)}, B^{(\ell)}$ are the system matrices for the $\ell$-th mode. The switching allows us to change the structure of the system, to break synchronization and to change the order of events. Note that each mode $\ell$ corresponds to a set of required synchronizations and an event order schedule, which leads to a model (2) with system matrices $\left(A^{(\ell)}, B^{(\ell)}\right)$ for the $\ell$-th model.

The moments of switching are determined by a switching mechanism. We define the switching variable $z(k)$, which may depend on the previous state $x(k-1)$, previous mode $\ell(k-1)$, the input variable $u(k)$ and an (additional) control variable $v(k)$ :

$z(k)=\Phi(x(k-1), \ell(k-1), u(k), v(k)) \in \mathbb{R}^{n_{z}}$.

We partition $\mathbb{R}^{n_{z}}$ in $n_{\mathrm{m}}$ subsets $\mathcal{Z}^{(i)}, i=$ $1, \ldots, n_{\mathrm{m}}$. The mode $\ell(k)$ is now obtained by determining in which set $z(k)$ is for event $k$. So if $z(k) \in \mathcal{Z}^{(i)}$, then $\ell(k)=i$. In some systems the switching mechanism will completely depend on the state $x(k-1)$ and input $u(k)$, in other examples $z(k)$ will be equal to $v(k)$ and so we can control the switching by choosing an appropriate $v(k)$.

\section{EXAMPLES OF SWITCHING MAX-PLUS-LINEAR SYSTEMS}

\subsection{Production system}

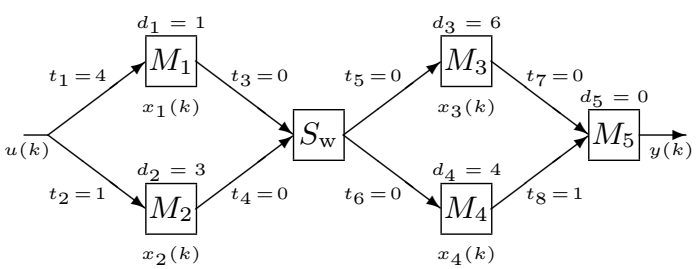

Figure 1: A production system.

Consider the production system of Figure 1. This system consists of five machines $M_{1}, M_{2}, M_{3}, M_{4}$ and $M_{5}$. The raw material is fed to machine $M_{1}$ and $M_{2}$, where preprocessing is done. Both products now have to be finished in either unit $M_{3}$ and $M_{4}$, which basically perform the same task, but the processing time of $M_{3}$ is longer than $M_{4}$. Therefore, the products coming from machine $M_{1}$ and
$M_{2}$ are directed to a switching device $S_{\mathrm{w}}$, that feeds the first product in the $k$ th cycle to the slower machine $M_{3}$ and the second product to the faster machine $M_{4}$. Finally, the products are assembled (instantaneously ${ }^{1}$ ) in machine $M_{5}$ and become available. We assume that each machine starts working as soon as possible on each batch, i.e., as soon as the raw material or the required intermediate products are available, and as soon as the machine is idle (i.e., the previous batch has been finished and has left the machine). We define $u(k)$ as the time instant at which the system is fed for the $k$ th time, $y(k)=x_{5}(k)$ as time instant at which the $k$ th product leaves the system and $x_{i}(k)$ as the time instant at which machine $i$ starts for the $k$ th time. The variable $t_{j}$ for $j=1, \ldots, 4$ is the transportation time, and $d_{i}$ for $i=1, \ldots, 5$ is the processing time on machine $i$. The system equations for $x_{1}$ and $x_{2}$ are given by

$$
\begin{aligned}
& x_{1}(k)=\max \left(x_{1}(k-1)+d_{1}, u(k)+t_{1}\right) \\
& x_{2}(k)=\max \left(x_{2}(k-1)+d_{2}, u(k)+t_{2}\right)
\end{aligned}
$$

If $x_{1}(k)+d_{1} \leq x_{2}(k)+d_{2}$, the product of $M_{1}$ will be directed to $M_{3}$ and the product of $M_{2}$ will be directed to $M_{4}$, and we obtain:

$$
\begin{aligned}
x_{3}(k)= & \max \left(x_{1}(k)+d_{1}, x_{3}(k-1)+d_{3}\right) \\
= & \max \left(x_{1}(k-1)+2 d_{1}, x_{3}(k-1)+d_{3},\right. \\
& \left.u(k)+d_{1}+t_{1}\right) \\
x_{4}(k)= & \max \left(x_{2}(k)+d_{2}, x_{4}(k-1)+d_{4}\right) \\
= & \max \left(x_{2}(k-1)+2 d_{2}, x_{4}(k-1)+d_{4},\right. \\
& \left.u(k)+d_{2}+t_{2}\right) \\
x_{5}(k)= & \max \left(x_{3}(k)+d_{3}, x_{4}(k)+d_{4}+t_{8}\right) \\
= & \max \left(x_{1}(k-1)+2 d_{1}+t_{1}+d_{3},\right. \\
& x_{2}(k-1)+2 d_{2}+d_{4}+t_{8}, \\
& x_{3}(k-1)+2 d_{3}, x_{4}(k-1)+2 d_{4}+t_{8}, \\
& u(k)+d_{1}+t_{1}+d_{3}, \\
& \left.u(k)+d_{2}+t_{2}+d_{4}+t_{8}\right)
\end{aligned}
$$

For this first mode $\left(x_{1}(k)+d_{1} \leq x_{2}(k)+d_{2}\right)$ we obtain the system matrices

$$
A^{(1)}=\left[\begin{array}{ccccc}
1 & \varepsilon & \varepsilon & \varepsilon & \varepsilon \\
\varepsilon & 3 & \varepsilon & \varepsilon & \varepsilon \\
1 & \varepsilon & 6 & \varepsilon & \varepsilon \\
\varepsilon & 6 & \varepsilon & 4 & \varepsilon \\
8 & 11 & 12 & 9 & \varepsilon
\end{array}\right] \quad B^{(1)}=\left[\begin{array}{c}
4 \\
1 \\
5 \\
4 \\
11
\end{array}\right]
$$

Similarly, for this second mode $\left(x_{1}(k)+d_{1}>\right.$ $\left.x_{2}(k)+d_{2}\right)$ we obtain the system matrices

$$
A^{(2)}=\left[\begin{array}{ccccc}
1 & \varepsilon & \varepsilon & \varepsilon & \varepsilon \\
\varepsilon & 3 & \varepsilon & \varepsilon & \varepsilon \\
\varepsilon & 6 & 6 & \varepsilon & \varepsilon \\
1 & \varepsilon & \varepsilon & 4 & \varepsilon \\
7 & 12 & 12 & 9 & \varepsilon
\end{array}\right] \quad B^{(2)}=\left[\begin{array}{c}
4 \\
1 \\
4 \\
5 \\
10
\end{array}\right]
$$

\footnotetext{
${ }^{1}$ I.e. with a negligible processing time.
} 
To decide the switching mechanism, we define the switching variable

$$
\begin{aligned}
& {\left[\begin{array}{l}
z_{1}(k) \\
z_{2}(k)
\end{array}\right]=\left[\begin{array}{l}
x_{1}(k)+d_{1} \\
x_{2}(k)+d_{2}
\end{array}\right]} \\
& \quad=\left[\begin{array}{l}
\max \left(x_{1}(k-1)+2 d_{1}, u(k)+d_{1}+t_{1}\right) \\
\max \left(x_{2}(k-1)+2 d_{2}, u(k)+d_{2}+t_{2}\right)
\end{array}\right] \\
& =\left[\begin{array}{l}
\max \left(x_{1}(k-1)+2, u(k)+5\right) \\
\max \left(x_{2}(k-1)+6, u(k)+4\right)
\end{array}\right]
\end{aligned}
$$

and the sets

$$
\begin{aligned}
& \mathcal{Z}^{(1)}=\left\{z \in \mathbb{R}^{2} \mid z_{1} \leq z_{2}\right\} \\
& \mathcal{Z}^{(2)}=\left\{z \in \mathbb{R}^{2} \mid z_{1}>z_{2}\right\} .
\end{aligned}
$$

Now the state space equation of our system is given by (2).

\subsection{Railway network}

Consider the railroad network of Figure 2 (see also [11]). There are 4 stations in this railroad network (A, B, C and D) that are connected by 5 single tracks $(1 / 7,2 / 4,3,5,9)$ and one double track (tracks $6 \&$ 8). There are three trains available. The first train follows the route $D \rightarrow A \rightarrow B \rightarrow D$, the second train follows the route $A \rightarrow B \rightarrow C \rightarrow A$, and the third train follows the route $D \rightarrow A \rightarrow C \rightarrow D$. We assume that there exists a periodic timetable that schedules the earliest departure times of the trains. The period of the timetable is $T=60$ minutes. So if a departure of a train from station B is scheduled at 5.30 a.m., then there is also scheduled a departure of a train from station B at 6.30 a.m., 7.30 a.m., and so on.

Each track of the railway network has a number and a train allocated to it. For the sake of simplicity we will say "(virtual) train $j$ " to denote the (physical) train on a specific track. The number of tracks in the network is equal to 7 , the number of physical trains in the network is equal to 3 , and the number of virtual trains in the network is equal to 9 . Let $x_{j}(k)$, $j=1, \ldots, 9$ be the time instant at which train $j$ departs from its station for the $k$ th time. Let $d_{j}(k)$ be the departure time for this train according to the time schedule, and let $a_{j}(k)$ be the transportation time for this train $j$.

Table 1 summarizes the information in connection with the nominal travelling times and the departure times. All the times are measured in minutes. The indicated departure times are the earliest departure times in the initial station of the track expressed in minutes after the hour. The first period starts at time $t=0$. At the beginning of the first period the first train is in station A and the second train is in station

\begin{tabular}{|c|c|c|c|c|}
\hline train & $\begin{array}{l}\text { from } \\
\text {-to }\end{array}$ & $\begin{array}{l}\text { travel } \\
\text { time }\end{array}$ & $\begin{array}{l}\text { dep } \\
\text {-arr }\end{array}$ & constraints \\
\hline 1 & D-A & 12 & $00-12$ & $\begin{array}{l}\text { same train as } 3^{-} \\
\text {connects to } 9^{-} \\
\text {follows } 7^{-}\end{array}$ \\
\hline 2 & A-B & 12 & $15-27$ & $\begin{array}{l}\text { same train as } 1 \\
\text { connects to } 6^{-} \\
\text {follows } 4^{-}\end{array}$ \\
\hline 3 & B-D & 20 & $30-50$ & same train as 2 \\
\hline 4 & A-B & 12 & $19-31$ & $\begin{array}{l}\text { same train as } 6^{-} \\
\text {follows } 2 \\
\text { connects to } 7\end{array}$ \\
\hline 5 & B-C & 10 & $34-44$ & same train as 4 \\
\hline 6 & C-A & 25 & $47-12$ & same train as 5 \\
\hline 7 & D-A & 12 & $04-16$ & $\begin{array}{l}\text { same train as } 9^{-} \\
\text {follows } 1\end{array}$ \\
\hline 8 & A-C & 25 & $19-44$ & same train as 7 \\
\hline 9 & C-D & 10 & $47-57$ & $\begin{array}{l}\text { same train as } 8 \\
\text { connects to } 5\end{array}$ \\
\hline
\end{tabular}
B.

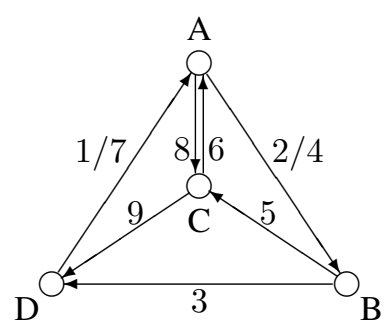

Figure 2: The railroad network of the example of Subsection 3.2 .

Note: $3^{-}$denotes train 3 in the previous cycle

Table 1: The nominal travelling times and the departure times for the railroad network.

The continuity constraints are that the trains on tracks 1, 2 and 3 are physically the same train, and the same holds for the trains on tracks 4, 5 and 6 and for the trains on tracks 7,8 and 9 .

Connection constraints are introduced to allow the passengers to change trains. In this network, train 1 has to wait for train 9 in the previous cycle with minimum connection time $c^{\min }=3$. In the same way, train 2 waits for train 6 in the previous cycle, train 4 wait for train 7 , and train 9 waits for train 5 . The minimum stopping time of train $j$ at station $j$ to allow passenger to get off or on the train is fixed at $s^{\min }=1$.

Follow constraints are introduced to guarantee sufficient separation time between two trains on the same track (moving in the same direction). In this network, train 4 is scheduled behind train 2 (train 4 follows train 2 ) with a minimum separation time 
$f^{\text {min }}=4$. In the same way, train 2 follows train 4 in the previous cycle, train 7 follows train 1, and train 1 follows train 7 in the previous cycle.

Each train departs as soon as all the connections are guaranteed (except for a connection when it is broken), the passengers have gotten the opportunity to change over and the earliest departure time indicated in the timetable has passed. We assume that in the first period all the trains depart according to schedule. The $j$-th state $x_{j}(k)$ is the time instant at which the train on track $j$ departs from the initial station of the track for the $k$ th time.

Now we write down the equations that describe the evolution of the $x_{j}(k)$ 's. First we consider the train on track 1 and we determine $x_{1}(k)$, the time instant at which this train departs from station A for the $k$ th time. The train has to wait at least until the train has arrived in station $\mathrm{A}$ for the $(k-1)$ th time $^{2}$ and the passengers have got the time to get out of the train so we have $x_{1}(k) \geq x_{3}(k-1)+a_{3}(k-1)+$ 1. Furthermore, the train on track 1 has to wait for the passengers of the train on track 9 in the $(k-$ $1)$ th cycle, which arrives in station $B$ at time instant $x_{9}(k-1)+a_{9}(k-1)$. The passengers have $c^{\min }=3$ minutes to change trains. Further the train on track 1 has to follow the train on track 7 in the previous cycle with a minimum separation time $f^{\text {min }}=4$. According to the timetable ${ }^{3}$ the train on track 1 can only depart after time instant $00+k 60$. Hence, we have

$$
\begin{gathered}
x_{1}(k)=\max \left(x_{3}(k-1)+a_{3}(k-1)+s^{\min },\right. \\
x_{7}(k-1)+f^{\min }, \\
\left.x_{9}(k-1)+a_{9}(k-1)+c^{\min }, d_{1}(k)\right) \\
=\max \left(x_{3}(k-1)+21, x_{7}(k-1)+4,\right. \\
\left.x_{9}(k-1)+13, k 60\right)
\end{gathered}
$$

for $k=1,2, \ldots$ with $x_{3}(0)=x_{9}(0)=-\infty$.

Using a similar reasoning, we find that the other departure times are given by

$$
\begin{aligned}
x_{2}(k)= & \max \left(x_{1}(k)+13, x_{4}(k-1)+4,\right. \\
& \left.x_{6}(k-1)+28,15+k 60\right) \\
x_{3}(k)= & \max \left(x_{2}(k)+13,30+k 60\right) \\
x_{4}(k)= & \max \left(x_{2}(k)+4, x_{6}(k-1)+26,\right.
\end{aligned}
$$

\footnotetext{
${ }^{2}$ Under nominal operations the $k$ th train on track 1 (e.g., the one that departs from station $D$ at 10.00 a.m.) proceeds to the $(k-1)$ th train on track 3 (which has departed from station $B$ at 9.30 a.m.) and not to the $k$ th train on track 3 (which will depart from station $B$ at 10.30 a.m.).

${ }^{3}$ Note that in a undisturbed, well-defined time schedule the term $d_{i}(k)$ will be the largest. However, if due to unforeseen circumstances (an incident, a late departure, etc.) one of the trains has a delay, the corresponding term can become larger than the others, train $i$ will depart later than the scheduled departure time $d_{i}(k)$ and will therefore also be delayed.
}

$$
\begin{aligned}
& \left.\qquad x_{7}(k)+15,19+k 60\right) \\
& x_{5}(k)=\max \left(x_{4}(k)+13,34+k 60\right) \\
& x_{6}(k)=\max \left(x_{5}(k)+11,47+k 60\right) \\
& x_{7}(k)=\max \left(x_{9}(k-1)+11, x_{1}(k)+4,4+k 60\right) \\
& x_{8}(k)=\max \left(x_{7}(k)+13,19+k 60\right) \\
& x_{9}(k)=\max \left(x_{8}(k)+26, x_{5}(k)+13,47+k 60\right) \\
& \text { for } k=1,2, \ldots \text { with } x_{j}(0)=-\infty \text { for } j= \\
& 1,2, \ldots, 9 .
\end{aligned}
$$

Using successive substitution we can eliminate all right-hand terms with index $k$. By defining the appropriate matrix $A^{(1)}$ and by using the $(\otimes, \oplus)$ notation, we can rewrite the state equations as:

$$
x(k)=A^{(1)}(k) \otimes x(k-1) \oplus d(k) .
$$

In the nominal operation we have assumed that some trains should give pre-defined connections to other trains, and the order of trains on the same track is fixed. However, if one of the preceding trains has a too large delay, then it is sometimes better — from a global performance viewpoint — to let a connecting train depart anyway or to change the departure order on a specific track. This is done in order to prevent an accumulation of delays in the network. In this paper we consider the switching between different operation modes, where each mode corresponds to a different set of pre-defined or broken connections and a specific order of train departures. We allow the system to switch between different modes, allowing us to break train connections and to change the order of trains. Note that any broken connection or change of train order leads to a new model, similar to the nominal equation (4), but now with adapted system matrix $A^{(\ell)}$ for the $\ell$-th model. We have the following system equation for the perturbed operation for $\ell=2, \ldots, n_{\mathrm{m}}$ :

$$
x(k)=A^{(\ell)}(k) \otimes x(k-1) \oplus d(k) .
$$

In this railway network the switching variable $z(k)$ is equal to the control vector $v(k)$, and each entry of $v(k)$ corresponds to a specific control action, so a specific (scheduled) synchronization or specific (scheduled) event order. We assume $v(k)$ to be binary, where $v_{i}(k)=0$ corresponds to the nominal case, and $v_{i}(k)=1$ to the perturbed case (the synchronization is broken or the order of two events is switched). Each combination $v_{1}(k), \ldots, v_{m}(k)$ corresponds to a fixed routing schedule with a specific train order and specific connections.

\section{THE MODEL PREDICTIVE CONTROL PROBLEM}

Consider switching max-plus-linear model (2)-(3). We have two possible input signals, $v(k)$ and $u(k)$. 
Let $\mathcal{V}(k)$ and $\mathcal{U}(k)$ be the sets of possible future control actions $v(k)$ and $u(k)$, respectively. Sometimes values are predefined (e.g. in the railway system $u(k)=d(k))$ or not applicable (e.g. in the production system $v(k)$ is not used). Often $v(k)$ is assumed to be binary, and each entry corresponds to a specific control action (e.g. a specific scheduled synchronization or specific scheduled event order).

Just as in conventional Model Predictive Control (MPC) [10] we define a cost criterion $J$ and we aim at computing the optimal input sequences $u(k), \ldots, u\left(k+N_{\mathrm{p}}-1\right), v(k), \ldots, v\left(k+N_{\mathrm{p}}-1\right)$ that minimize a cost criterion $J(k)$, possibly subject to linear constraints on the inputs and the states, where $N_{\mathrm{p}}$ is the prediction horizon. The cost criterion reflects the input and output cost functions ( $J_{\text {in }}$ and $J_{\text {out }}$, respectively) in the event period $\left[k, k+N_{\mathrm{p}}-1\right]$ :

$$
J(k)=J_{\text {out }}(k)+\lambda J_{\text {in }}(k),
$$

where $\lambda$ is a weighting parameter. The output cost function is usually chosen as

$$
J_{\text {out }}(k)=\sum_{j=0}^{N_{\mathrm{p}}-1}\|\hat{e}(k+j)\|,
$$

where $\|\cdot\|$ is an appropriate norm and $\hat{e}$ is the due date error (e.g. for the production system the due date error of a product is given by $\hat{e}_{i}(k)=$ $\max \left(y_{i}(k)-r_{i}(k), 0\right)$, where $r(k)$ is the desired due date of the product, and for the railway system the due date error is equal to the delay of a train, so $\left.\hat{e}_{i}(k)=\max \left(x_{i}(k)-d_{i}(k), 0\right)\right)$. The input cost function consists of two parts, $J_{\text {in }}=J_{\text {in }, u}+J_{\text {in }, v}$. The first part $J_{\text {in }, u}$ depends on $u(k+j), j=$ $k, \ldots, k+N_{\mathrm{p}}-1$ and is usually chosen as

$$
J_{\mathrm{in}, u}(k)=-\sum_{j=0}^{N_{\mathrm{p}}-1}\|u(k+j)\|,
$$

(see also [6]). The second part $J_{\text {in }, v}$ is a function of $v(k+j), j=k, \ldots, k+N_{\mathrm{p}}-1$. For different applications, $J_{\text {in }, v}$ will have different appearances (e.g. for the production system $J_{\mathrm{in}, v}(k)=0$ and for the railway system we choose $J_{\mathrm{in}, v}(k)=$ $\left.\sum_{j=0}^{N_{\mathrm{p}}-1} \sum_{i=1}^{n_{v}} v_{i}(k+j)\right)$.

Since the input signal $u(k)$ correspond to consecutive event occurrence times, we have the additional condition for $j=0, \ldots, N_{\mathrm{p}}-1$ :

$$
\Delta u(k+j)=u(k+j)-u(k+j-1) \geq 0 .
$$

Furthermore, in order to reduce the number of decision variables and the corresponding computational complexity we introduce a control horizon
$N_{\mathrm{c}}\left(\leq N_{\mathrm{p}}\right)$ and we impose the additional condition that the input rate should be constant from the point $k+N_{\mathrm{c}}-1$ on, so

$$
\Delta u(k+j)=\Delta u\left(k+N_{\mathrm{c}}-1\right),
$$

for $j=N_{\mathrm{c}}, \ldots, N_{\mathrm{p}}-1$. The same often holds for the control variable $v(k)$, which will be assumed to be constant beyond control horizon $N_{\mathrm{c}}$. This results in the constraint $v(k+j)=v\left(k+N_{\mathrm{c}}-1\right)$ for $j=N_{\mathrm{c}}, \ldots, N_{\mathrm{p}}-1$, or equivalently

$$
\Delta v(k+j)=v(k+j)-v(k+j-1)=0,
$$

for $j=N_{\mathrm{c}}, \ldots, N_{\mathrm{p}}-1$. Now the MPC control problem for event step $k$ can be defined as:

$$
\min _{\{u(k) \in \mathcal{U}, v(k) \in \mathcal{V}(k)\}} J(k)
$$

subject to

$$
\begin{aligned}
& \quad x(k+j)=A^{(\ell(k))}(k) \otimes x(k+j-1) \\
& \qquad B^{(\ell(k))}(k) \otimes u(k+j) \\
& \Phi(x(k-1), \ell(k-1), u(k), v(k)) \in \mathcal{Z}^{(\ell(k))} \\
& \Delta u(k+j) \geq 0 \\
& \Delta v(k+m)=0 \\
& \quad \Delta u(k+m)-\Delta u\left(k+N_{\mathrm{c}}-1\right)=0 \\
& A_{\mathrm{c}}(k) \tilde{u}(k)+B_{\mathrm{c}}(k) \tilde{y}(k) \leq c_{\mathrm{c}}(k) \\
& \text { for } j=0, \ldots, N_{\mathrm{p}}-1, \quad m=N_{\mathrm{c}}, \ldots, N_{\mathrm{p}}-1
\end{aligned}
$$

where (13) may represent additional linear constraints on the inputs and the outputs.

MPC uses a receding horizon principle. This means that after computation of the optimal control sequences $u(k), \ldots, u\left(k+N_{\mathrm{c}}-1\right)$ and $v(k), \ldots, v\left(k+N_{\mathrm{c}}-1\right)$, only the first control samples $u(k)$ and $v(k)$ will be implemented, subsequently the horizon is shifted one sample, and the optimization is restarted with new information of the measurements.

In principle we have all elements to solve the receding horizon control problem (7)-(13). In general we will have a mixed integer optimal control problem with both real parameters and binary parameters. Sometimes (e.g. the production system) the problem can be recast as a Extended Linear Complementary Problem (ELCP) and can be solved efficiently [7]. If the optimization is over a binary valued $v(k)$ (e.g. the railway problem) we obtain an integer optimization problem, which can be solved using genetic algorithms [5], tabu search [9], or a branch-and-bound method [3]. In some particular cases the problem can be recast as a Mixed Integer Linear Programming (MILP) or a Mixed Integer Quadratic Programming (MIQP), for which reliable algorithms are available $[2,8]$. 
The application of the derived controller design method to a railway network is given in [11].

\section{DISCUSSION}

We have presented a new way to model a class of discrete event systems - the max-plus-linear discrete event systems - that can operate in different modes, in which the dynamics can be described by a model that is "linear" in the max-plus algebra. We have discussed two examples, a production system and a railway network. An MPC controller design technique has been derived for this type of systems. In general the resulting optimization problem requires a mixed integer optimization algorithm.

In future research we will study on the characterization of all discrete event systems that can be recast as a switching max-plus-linear system. Furthermore, we will try to find out what conditions are needed on $J, \Phi$ and $\mathcal{Z}^{(\ell(k))}$ to obtain particular optimization problems (ELCP, MILP, MIQP).

\section{Acknowledgments}

Research partially funded by the Dutch Technology Foundation STW project "Model predictive control for hybrid systems" (DMR.5675), by the European IST project "Modelling, Simulation and Control of Nonsmooth Dynamical Systems (SICONOS)" (IST2001-37172), and by the NWO/STW VIDI project "Multi-agent control of large-scale hybrid systems" (DWV.6188).

\section{References}

[1] F. Baccelli, G. Cohen, G.J. Olsder, and J.P. Quadrat. Synchronization and Linearity. John Wiley \& Sons, New York, 1992.

[2] A. Bemporad and M. Morari. Control of integrated logic, dynamics, and constraints. Automatica, 35(3):407-427, 1999.

[3] C. Cordier, H. Marchand, R. Laundy, and L.A. Wolsey. bc-opt: A branch-and-cut code for mixed integer programs. Mathematical Programming, Series A, 86(2):335-353, 1999.

[4] R.A. Cuninghame-Green. Minimax Algebra, volume 166 of Lecture Notes in Economics and Mathematical Systems. Springer-Verlag, Berlin, Germany, 1979.

[5] L. Davis, editor. Handbook of Genetic Algorithms. Van Nostrand Reinhold, New York, 1991.

[6] B. De Schutter and T.J.J. van den Boom. Model predictive control for max-plus-linear dis- crete event systems. Automatica, 37(7):1049-1056, July 2001.

[7] B. De Schutter and T.J.J. van den Boom. Model predictive control for max-min-plus-scaling systems - efficient implementation. In WODES 2002, Zaragossa, Spain, pages 343-348, 2002.

[8] R. Fletcher and S. Leyffer. Numerical experience with lower bounds for MIQP branch-andbound. SIAM Journal on Optimization, 8(2):604616, 1998.

[9] F. Glover and M. Laguna. Tabu Search. Kluwer Academic Publishers, Boston, 1997.

[10] J.M. Maciejowski. Predictive control with constraints. Prentice Hall, Pearson Education Limited, Harlow, UK, 2002.

[11] T.J.J. van den Boom and B. De Schutter. Modelling and control of railway networks. In American Control Conference 2004, Boston, MA, USA, 2004. 